\title{
Log-Harnack inequality for Gruschin type semigroups
}

\author{
Feng-Yu Wang and Lihu Xu
}

\begin{abstract}
By constructing a coupling in two steps and using the Girsanov theorem under a regular conditional probability, the log-Harnack inequality is established for a large class of Gruschin type semigroups whose generator might be both degenerate and non-Lipschitz.
\end{abstract}

\section{Introduction}

In recent years, regularity estimates has been investigated for some typical subelliptic diffusion semigroups; see [7], [22], [24] for the study of generalized stochastic Hamiltonian systems, and see [2], [5], [6], [8] for gradient estimates and Harnack inequalities on Heisenberg groups. This paper aims to investigate the log-Harnack inequality introduced in [13], [15] for Gruschin type semigroups whose generators are degenerate and possibly singular. This inequality is a weaker version of the dimension-free Harnack inequality introduced in [14], and has a number of applications to heat kernel estimates and transportation-cost inequalities; see, e.g., Section 4 in [18].

Let us start with the classical Gruschin semigroup on $\mathbb{R}^{2}$ of order $l>0$, that is generated by

$$
L\left(x^{(1)}, x^{(2)}\right):=\frac{1}{2}\left(\frac{\partial^{2}}{\partial\left(x^{(1)}\right)^{2}}+\left|x^{(1)}\right|^{2 l} \frac{\partial^{2}}{\partial\left(x^{(2)}\right)^{2}}\right) .
$$

The corresponding diffusion process can be constructed by solving the SDE

$$
\left\{\begin{array}{l}
\mathrm{d} X_{t}^{(1)}=\mathrm{d} B_{t}^{(1)} \\
\mathrm{d} X_{t}^{(2)}=\left|X_{t}^{(1)}\right|^{l} \mathrm{~d} B_{t}^{(2)}
\end{array}\right.
$$

where $B_{t}:=\left(B_{t}^{(1)}, B_{t}^{(2)}\right)$ is a two-dimensional Brownian motion. Clearly, the equation is degenerate, and when $l<1$ the coefficient in the second equation is

Mathematics Subject Classification (2010): Primary 60J75; Secondary 60J45.

Keywords: Gruschin semigroup, log-Harnack inequality, coupling, regular conditional probability. 
non-Lipschitz. In the simplest case that $l=1$, the generalized curvature-dimension condition introduced in [4] holds, so that the gradient estimates and Harnack inequalities derived in [3], [4] are valid for the associated semigroup. When $l$ is a natural number larger than 1, a more general version of the curvature condition has been confirmed in [18], which also implies explicit gradient estimates for the semigroup. Moreover, for general $l \geq 1$, a Bismut type derivative formula was derived for the semigroup in [17] by using the Malliavin calculus. However, due to the singularity of the coefficient, the arguments used in these papers are not valid if $l \in(0,1)$, and except for $l=1$, the validity of the log-Harnack inequality for the semigroup is not yet established. In this paper we aim to establish the log-Harnack inequality for the Gruschin semigroup for all $l>0$. However, the argument used here does not imply the dimension-free Harnack inequality in the sense of [14] for the Gruschin semigroup.

A key tool in the study is the method of coupling by change of measure introduced in [1]. This method has been developed and applied to various finite and infinite-dimensional models; see, e.g., [7], [9], [10], [11], [12], [16], [20], [19], $[21],[23]$ and references therein. In the study we have to overcome new difficulties due to the high degeneracy (for large $l$ ) and the singularity (for small $l$ ) of the coefficient.

We consider the following more general SDE for $X_{t}:=\left(X_{t}^{(1)}, X_{t}^{(2)}\right)$ on $\mathbb{R}^{m} \times$ $\mathbb{R}^{d}=\mathbb{R}^{m+d}(m, d \geq 1)$ :

$$
\left\{\begin{array}{l}
\mathrm{d} X_{t}^{(1)}=b^{(1)}\left(t, X_{t}^{(1)}\right) \mathrm{d} t+\sigma^{(1)}(t) \mathrm{d} B_{t}^{(1)}, \\
\mathrm{d} X_{t}^{(2)}=b^{(2)}\left(t, X_{t}\right) \mathrm{d} t+\sigma^{(2)}\left(t, X_{t}^{(1)}\right) \mathrm{d} B_{t}^{(2)},
\end{array}\right.
$$

where $B_{t}:=\left(B_{t}^{(1)}, B_{t}^{(2)}\right)$ is the $(m+d)$-dimensional Brownian motion on a complete probability space $(\Omega, \mathcal{F}, \mathbb{P})$ with natural filtration $\left\{\mathcal{F}_{t}\right\}_{t \geq 0}$, and

$$
\begin{aligned}
& b^{(1)}:[0, \infty) \times \mathbb{R}^{m} \rightarrow \mathbb{R}^{m}, \quad b^{(2)}:[0, \infty) \times \mathbb{R}^{m+d} \rightarrow \mathbb{R}^{d}, \\
& \sigma^{(1)}:[0, \infty) \rightarrow \mathbb{R}^{m} \otimes \mathbb{R}^{m}, \quad \sigma^{(2)}:[0, \infty) \times \mathbb{R}^{m} \rightarrow \mathbb{R}^{d} \otimes \mathbb{R}^{d}
\end{aligned}
$$

are measurable, and $b^{(1)}, b^{(2)}$ and $\sigma^{(2)}$ are continuous in the second variable. Assume:

(A.1) There exists a decreasing function $\lambda:[0, \infty) \rightarrow(0, \infty)$ such that

$$
\sigma^{(1)}(t) \sigma^{(1)}(t)^{*} \geq \lambda_{t}^{2} I_{m \times m}, \quad t \geq 0
$$

(A.2) There exists an increasing function $K:[0, \infty) \rightarrow \mathbb{R}$ such that

$$
\left\langle b^{(1)}\left(t, x^{(1)}\right)-b^{(1)}\left(t, y^{(1)}\right), x^{(1)}-y^{(1)}\right\rangle \leq K_{t}\left|x^{(1)}-y^{(1)}\right|^{2}, t \geq 0, x^{(1)}, y^{(1)} \in \mathbb{R}^{m} .
$$

(A.3) There exist increasing functions $\Theta:[0, \infty) \rightarrow \mathbb{R}, h:[0, \infty) \rightarrow[1, \infty)$, and $\varphi .:[0, \infty)^{2} \rightarrow[0, \infty)$ with $\varphi(0)=0$ such that

$$
\begin{aligned}
\left\langle b^{(2)}(t, x)-b^{(2)}(t, y)\right. & \left., x^{(2)}-y^{(2)}\right\rangle+\frac{1}{2}\left\|\sigma^{(2)}\left(t, x^{(1)}\right)-\sigma^{(2)}\left(t, y^{(1)}\right)\right\|_{H S}^{2} \\
& \leq \Theta_{t}\left|x^{(2)}-y^{(2)}\right|^{2}+\varphi_{t}\left(\left|x^{(1)}-y^{(1)}\right|^{2}\right) h\left(\left|x^{(1)}\right| \vee\left|y^{(1)}\right|\right)
\end{aligned}
$$

holds for all $t \geq 0$ and $x=\left(x^{(1)}, x^{(2)}\right), y=\left(y^{(1)}, y^{(2)}\right) \in \mathbb{R}^{m+d}$. 
It is well known that (A.1) implies the existence, uniqueness, and non-explosion of strong solutions to the first equation in (1.1). Once $X_{t}^{(1)}$ is fixed, then it follows from (A.3) that the second equation in (1.1) admits a unique global solution. Note that (A.3) allows $\sigma^{(2)}(t, \cdot)$ to be merely Hölder continuous when, e.g., $\varphi_{t}(r)=r^{\alpha}$ for some constant $\alpha \in(0,1)$. For any $x=\left(x^{(1)}, x^{(2)}\right) \in \mathbb{R}^{m+d}$, we let $X_{t}(x)=$ $\left(X_{t}^{(1)}(x), X_{t}^{(2)}(x)\right)$ denote the solution to (1.1) with $X_{0}=x$. Since $X_{t}^{(1)}(x)$ does not depend on $x^{(2)}$ we also write $X_{t}^{(1)}(x)=X_{t}^{(1)}\left(x^{(1)}\right)$. We intend to establish Harnack type inequalities for the associated semigroup $P_{t}$ :

$$
P_{t} f(x):=\mathbb{E} f\left(X_{t}(x)\right), \quad f \in \mathcal{B}_{b}\left(\mathbb{R}^{m+d}\right), \quad t \geq 0, x \in \mathbb{R}^{m+d} .
$$

We remark that (A.1) means that the first component process $X_{t}^{(1)}$ is a nondegenerate diffusion process on $\mathbb{R}^{m},(\mathrm{~A} .2)$ is the usual semi-Lipschitz condition for this process, and when e.g. $b^{(2)}$ is independent of $x^{(1)}$ and semi-Lipschitz in $x^{(2)},(\mathrm{A} .3)$ holds provided

$$
\frac{1}{2}\left\|\sigma^{(2)}\left(t, x^{(1)}\right)-\sigma^{(2)}\left(t, y^{(1)}\right)\right\|_{H S}^{2} \leq \varphi_{t}\left(\left|x^{(1)}-y^{(1)}\right|^{2}\right) h\left(\left|x^{(1)}\right| \vee\left|y^{(1)}\right|\right) .
$$

In particular, for the Gruschin semigroup where $\sigma^{(2)}\left(t, x^{(1)}\right)=\left|x^{(1)}\right|^{l}$, this condition holds for $\varphi_{t}(r)=r^{l \wedge 1}$ and $h(r)=c \vee r^{(l-1)^{+}}$for some constant $c \geq 1$.

In order to control the degeneracy of $\sigma^{(2)}(t, \cdot)$, we need the condition (1.2)

$\psi_{T}\left(x^{(1)}, y^{(1)}\right):=\sup _{t \in[T, 2 T]} \mathbb{E}^{y^{(1)}}\left\{\left\|\sigma^{(2)}\left(t, X_{t}^{(1)}\right)^{-1}\right\|^{2} \sup _{s \in[0, T]} h\left(\left|X_{s}^{(1)}\right|+\left|x^{(1)}-y^{(1)}\right|\right)\right\}<\infty$

for $T>0$ and $x^{(1)}, y^{(1)} \in \mathbb{R}^{m}$, where $\mathbb{E}^{y^{(1)}}$ is the expectation of $X_{t}^{(1)}\left(y^{(1)}\right),\left\|\sigma^{-1}\right\|$ stands for the operator norm of the inverse of a $d \times d$-matrix $\sigma$, and when the matrix is not invertible we take $\left\|\sigma^{-1}\right\|=\infty$.

Theorem 1.1. Assume that (A.1), (A.2), (A.3), and (1.2) hold. Then for any strictly positive function $f \in \mathcal{B}_{b}\left(\mathbb{R}^{m+d}\right), x=\left(x^{(1)}, x^{(2)}\right), y=\left(y^{(1)}, y^{(2)}\right) \in \mathbb{R}^{m+d}$, and $T>0$,

$$
\begin{aligned}
P_{2 T} & \log f(y) \leq \log P_{2 T} f(x)+\frac{K_{T}\left|x^{(1)}-y^{(1)}\right|^{2}}{\lambda_{T}^{2}\left(1-\mathrm{e}^{-2 K_{T} T}\right)} \\
& +\frac{\Theta_{2 T} \mathrm{e}^{2 \Theta_{T} T} \psi_{T}\left(x^{(1)}, y^{(1)}\right)}{\mathrm{e}^{-2 \Theta_{2 T} T}-\mathrm{e}^{-4 \Theta_{2 T} T}}\left\{\left|x^{(2)}-y^{(2)}\right|^{2}+\frac{\left(1-\mathrm{e}^{-2 \Theta_{T} T}\right) \varphi_{T}\left(\left|x^{(1)}-y^{(1)}\right|^{2}\right)}{\Theta_{T}}\right\} .
\end{aligned}
$$

We return to the classical Gruschin semigroup for which $m=d=1, b^{(1)}=$ $b^{(2)}=0, \sigma^{(1)}=1$ and $\sigma^{(2)}\left(t, x^{(1)}\right)=\left|x^{(1)}\right|^{l}$. Then (A.1)-(A.3) hold for $\lambda=1$, $K=\Theta=0, \varphi(r)=r^{l \wedge 1}$ and $h(r)=c_{1} \vee r^{(l-1)^{+}}$for some constant $c_{1} \geq 1$. When $l \in(0,1 / 2)$, we may take $h \equiv 1$ so that

$$
\psi_{T}\left(x^{(1)}, y^{(1)}\right)=\sup _{t \in[T, 2 T]} \int_{\mathbb{R}} \frac{1}{|z|^{2 l} \sqrt{2 t \pi}} e^{-\left|z-x^{(1)}\right|^{2} /(2 t)} \mathrm{d} z \leq \frac{c_{2}}{T^{l}}<\infty
$$

for some constant $c_{2}>0$. 
Therefore, according to Theorem 1.1, the log-Harnack inequality

$P_{2 T} \log f(y) \leq \log P_{2 T} f(x)+\frac{\left|x^{(1)}-y^{(1)}\right|^{2}}{2 T}+\frac{c}{T^{l+1}}\left\{\left|x^{(2)}-y^{(2)}\right|^{2}+2 T\left|x^{(1)}-y^{(1)}\right|^{2 l}\right\}$

holds. On the other hand, it is easy to see that $\psi_{T}=\infty$ for $l \geq 1 / 2$. Similarly, for the Gruschin semigroup on $\mathbb{R}^{m+d}$, i.e., $b^{(1)}=0, b^{(2)}=0, \sigma^{(1)}=I_{m \times m}$ and $\sigma^{(2)}\left(x^{(1)}\right)=\left|x^{(1)}\right|^{l} I_{d \times d}, \psi_{T}\left(x^{(1)}, y^{(1)}\right)<\infty$ (and hence the log-Harnack inequality holds) if and only if $l \in(0, m / 2)$.

To derive the log-Harnack inequality for the Gruschin semigroup for all $l>0$, we relax the condition (1.2) by using the invertibility of the following integral matrix $Q_{T}$ to replace that of $\sigma^{(2)}$. To this end, we will need to assume that $b^{(2)}(t, x)$ is linear in $x^{(2)}$; that is, $b^{(2)}(t, x)=A x^{(2)}+\tilde{b}^{(2)}\left(t, x^{(1)}\right)$ for some $d \times d$-matrix $A$ and some $\tilde{b}^{(2)} \in C\left([0, \infty) \times \mathbb{R}^{m} ; \mathbb{R}^{d}\right)$. Let

$$
Q_{T}=\int_{T}^{2 T} \mathrm{e}^{A(T-t)} \sigma^{(2)}\left(t, X_{t}^{(1)}\right) \sigma^{(2)}\left(t, X_{t}^{(1)}\right)^{*} \mathrm{e}^{A^{*}(T-t)} \mathrm{d} t, \quad T>0 .
$$

Theorem 1.2. Assume that (A.1), (A.2), and (A.3) hold for $b^{(2)}(t, x)=A x^{(2)}+$ $\tilde{b}^{(2)}\left(t, x^{(1)}\right)$, where $A$ is a $d \times d$-matrix and $\tilde{b}^{(2)} \in C\left([0, \infty) \times \mathbb{R}^{m} ; \mathbb{R}^{d}\right)$. Let $\theta_{T}=$ $\sup _{t \in[0, T]}\left\|\mathrm{e}^{-A t}\right\|$. If $Q_{T}$ is invertible and

$$
\begin{aligned}
\Psi_{T}\left(x^{(1)}, y^{(1)}\right):=\mathbb{E}^{y^{(1)}}\left\{\left\|Q_{T}^{-1}\right\|^{2}\right. & \left(\int_{T}^{2 T}\left\|\sigma^{(2)}\left(t, X_{t}^{(1)}\right)\right\|^{2} \mathrm{~d} t\right) \\
& \left.\times \sup _{t \in[0, T]} h\left(\left|X_{t}^{(1)}\right|+\left|x^{(1)}-y^{(1)}\right|\right)\right\}<\infty,
\end{aligned}
$$

then for any strictly positive $f \in \mathcal{B}_{b}\left(\mathbb{R}^{m+d}\right)$,

$$
\begin{aligned}
& P_{2 T} \log f(y) \leq \log P_{2 T} f(x)+\frac{K_{T}\left|x^{(1)}-y^{(1)}\right|^{2}}{\lambda_{T}^{2}\left(1-\mathrm{e}^{-2 K_{T} T}\right)} \\
& \quad+\frac{\theta_{T} \mathrm{e}^{2 \Theta_{T} T} \Psi_{T}\left(x^{(1)}, y^{(1)}\right)}{2}\left\{\left|x^{(2)}-y^{(2)}\right|^{2}+\frac{1-\mathrm{e}^{-2 \Theta_{T} T}}{\Theta_{T}} \varphi_{T}\left(\left|x^{(1)}-y^{(1)}\right|^{2}\right)\right\} .
\end{aligned}
$$

Because of Theorem 1.2, we can prove the log-Harnack inequality for the Gruschin semigroup on $\mathbb{R}^{m+d}$ for any $l>0$. Of course, one may also construct more general examples to illustrate Theorem 1.2.

Corollary 1.3 (Gruschin Semigroup). Let $b^{(1)}=0, b^{(2)}=0, \sigma^{(1)}=I_{m \times m}$, and $\sigma^{(2)}\left(x^{(1)}\right)=\left|x^{(1)}\right|^{l} I_{d \times d}$ for some constant $l>0$. Then there exists a constant $c>0$ such that

$$
\begin{aligned}
& P_{2 T} \log f(y) \leq \log P_{2 T} f(x)+\frac{\left|x^{(1)}-y^{(1)}\right|^{2}}{2 T} \\
& \quad+\frac{c\left(\left|x^{(1)}\right|^{2(l-1)^{+}}+\left|y^{(1)}\right|^{2(l-1)^{+}}+T^{(l-1)^{+}}\right)\left(\left|x^{(2)}-y^{(2)}\right|^{2}+2 T\left|x^{(1)}-y^{(1)}\right|^{2(l \wedge 1)}\right)}{T^{l+1}}
\end{aligned}
$$

holds for all $T>0$ and $x, y \in \mathbb{R}^{m+d}$. 
In the next two sections, we prove Theorem 1.1, Theorem 1.2, and Corollary 1.3. In the proof of Theorem 1.1, the additional drifts constructed in the coupling are adapted so that the usual argument applies. However, in the proof of Theorem 1.2 the drift constructed for the coupling of the second component process is merely adapted under the conditional probability given $B^{(1)}$. A new trick is then introduced to derive the log-Harnack inequality.

\section{Proof of Theorem 1.1}

Let $x=\left(x^{(1)}, x^{(2)}\right), y=\left(y^{(1)}, y^{(2)}\right)$, and $T>0$ be fixed. The idea for establishing a Harnack type inequality of $P_{2 T}$ using a coupling by change of measure is as follows. Construct two processes $X_{t}$, and $Y_{t}$ and a probability density function $R$ such that $X_{2 T}=Y_{2 T}, X_{0}=x, Y_{0}=y$, and

$$
P_{2 T} f(x)=\mathbb{E} f\left(X_{2 T}\right), \quad P_{2 T} f(y)=\mathbb{E}\left\{R f\left(Y_{2 T}\right)\right\}, \quad f \in \mathcal{B}_{b}\left(\mathbb{R}^{m+d}\right) .
$$

Then, by the Young inequality, for strictly positive $f$ one obtains

$$
\begin{aligned}
P_{2 T} \log f(y) & =\mathbb{E}\left\{R \log f\left(Y_{2 T}\right)\right\}=\mathbb{E}\left\{R \log f\left(X_{2 T}\right)\right\} \\
& \leq \mathbb{E}(R \log R)+\log P_{2 T} f(x) .
\end{aligned}
$$

This implies the log-Harnack inequality provided $\mathbb{E}(R \log R)<\infty$.

When the SDE is driven by additive noise, this idea can be easily realized by adding a proper drift to the equation and using the Girsanov theorem. In the case of nondegenerate multiplicative noise, the argument has been modified in [16] by constructing a coupling with singular additional drifts. For the present model, as the SDE is driven by multiplicative noise with a possibly degenerate and singular coefficient, it is hard to follow the known ideas to construct a coupling in one step. What we will do in this paper is to construct a coupling in two steps, where the second step will be realized under the regular conditional probability given $B^{(1)}$ :

(1) We first construct a coupling $\left(X_{t}^{(1)}, Y_{t}^{(1)}\right)$ by change of measure for the first component of the process such that $X_{t}^{(1)}=Y_{t}^{(1)}$ for $t \geq T$. This part is now standard as the first equation in (1.1) is driven by the nondegenerate additive noise $\sigma^{(1)}(t) \mathrm{d} B_{t}^{(1)}$.

(2) Once $X_{t}^{(1)}=Y_{t}^{(1)}$ holds for $t \geq T$, the equations for $X_{t}^{(2)}$ and $Y_{t}^{(2)}$ will have the same noise term for $t \geq T$, so that we are able to construct a coupling by change of measure for them such that $X_{2 T}^{(2)}=Y_{2 T}^{(2)}$.

\subsection{Construction of the coupling}

Throughout this section, we assume that (A.1)-(A.3) and condition (1.2) hold. We first construct the Brownian motion $B_{t}$ as the coordinate process on the Wiener space $(\Omega, \mathcal{F}, \mathbb{P})$, where $\Omega=C\left([0, \infty) ; \mathbb{R}^{m+d}\right)=C\left([0, \infty) ; \mathbb{R}^{m}\right) \times C\left([0, \infty) ; \mathbb{R}^{d}\right), \mathcal{F}$ is the Borel $\sigma$-field, and $\mathbb{P}$ is the Wiener measure (that is, the distribution of the $(m+d)$-dimensional Brownian motion starting at 0$)$. 
Let

$$
B_{t}(\omega)=\left(B_{t}^{(1)}(\omega), B_{t}^{(2)}(\omega)\right)=\left(\omega_{t}^{(1)}, \omega_{t}^{(2)}\right), \quad \omega=\left(\omega^{(1)}, \omega^{(2)}\right) \in \Omega, \quad t \geq 0 .
$$

Then $B_{t}$ is the $(m+d)$-dimensional Brownian motion with respect to the natural filtration $\left(\mathcal{F}_{t}\right)_{t \geq 0}$. Let $\mathcal{F}^{(1)}=\sigma\left(B_{t}^{(1)}: t \geq 0\right)$ and $\mathcal{F}_{t}^{(2)}=\sigma\left(B_{s}^{(2)}: 0 \leq s \leq t\right), t \geq 0$. It is well known that the conditional regular probability $\mathbb{P}\left(\cdot \mid \mathcal{F}^{(1)}\right)$ given $\overline{\mathcal{F}}^{(1)}$ exists. This structure will enable us to first construct a coupling $\left(X_{t}^{(1)}, Y_{t}^{(1)}\right)$ for the first component process up to time $T$ under the probability $\mathbb{P}$, then construct a coupling $\left(X_{t}^{(2)}, Y_{t}^{(2)}\right)$ for the second component process from time $T$ under the regular conditional probability $\mathbb{P}\left(\cdot \mid \mathcal{F}^{(1)}\right)$. For any probability measure $\tilde{\mathbb{P}}$ on $(\Omega, \mathcal{F})$, we denote by $\mathbb{E}_{\tilde{\mathbb{P}}}$ the expectation with respect to $\tilde{\mathbb{P}}$. When $\tilde{\mathbb{P}}=\mathbb{P}$, we simply denote the expectation by $\mathbb{E}$ as usual.

Let $X_{t}=\left(X_{t}^{(1)}, X_{t}^{(2)}\right)$ solve the equation (1.1) with $X_{0}=x=\left(x^{(1)}, x^{(2)}\right)$. Given $Y_{0}=y=\left(y^{(1)}, y^{(2)}\right) \in \mathbb{R}^{m+d}$, we are going to construct $Y_{t}^{(1)}$ on $\mathbb{R}^{m}$ and $Y_{t}^{(2)}$ on $\mathbb{R}^{d}$ respectively, such that $Y_{t}^{(1)}=X_{t}^{(1)}$ for $t \geq T$ and $Y_{2 T}^{(2)}=X_{2 T}^{(2)}$.

2.1.1. Construction of $\boldsymbol{Y}_{t}^{(1)}$. Consider the equation

$$
\mathrm{d} Y_{t}^{(1)}=b^{(1)}\left(t, Y_{t}^{(1)}\right) \mathrm{d} t+\sigma^{(1)}(t) \mathrm{d} B_{t}^{(1)}-v_{t}^{(1)} \mathrm{d} t, \quad Y_{0}^{(1)}=y^{(1)},
$$

where

$$
v_{t}^{(1)}:=\frac{2 K_{T}\left|x^{(1)}-y^{(1)}\right| \mathrm{e}^{-K_{T} t}\left(Y_{t}^{(1)}-X_{t}^{(1)}\right)}{\left(1-\mathrm{e}^{-2 K_{T} T}\right)\left|X_{t}^{(1)}-Y_{t}^{(1)}\right|} 1_{\left\{X_{t}^{(1)} \neq Y_{t}^{(1)}\right\}}, \quad t \geq 0 .
$$

Obviously, the equation has a unique strong solution before the coupling time

$$
\tau_{1}:=\inf \left\{t \geq 0: X_{t}^{(1)}=Y_{t}^{(1)}\right\} .
$$

Then, letting $Y_{t}^{(1)}=X_{t}^{(1)}$ for $t \geq \tau_{1}$, we see that $\left(Y_{t}^{(1)}\right)_{t \geq 0}$ is a strong solution to $(2.2)$. So, we can reformulate $v_{t}^{(1)}$ as

$$
v_{t}^{(1)}=\frac{2 K_{T}\left|x^{(1)}-y^{(1)}\right| \mathrm{e}^{-K_{T} t}\left(Y_{t}^{(1)}-X_{t}^{(1)}\right)}{\left(1-\mathrm{e}^{-2 K_{T} T}\right)\left|X_{t}^{(1)}-Y_{t}^{(1)}\right|} 1_{\left[0, \tau_{1}\right)}(t), \quad t \geq 0 .
$$

Proposition 2.1. For any $t \geq 0$,

$$
\left|X_{t}^{(1)}-Y_{t}^{(1)}\right| \leq \frac{\mathrm{e}^{-K_{T} t}-\mathrm{e}^{-K_{T}(2 T-t)}}{1-\mathrm{e}^{-2 K_{T} T}}\left|x^{(1)}-y^{(1)}\right| 1_{[0, T]}(t) \leq\left|x^{(1)}-y^{(1)}\right| 1_{[0, T]}(t) .
$$

Consequently, $\tau_{1} \leq T$ and $X_{t}^{(1)}=Y_{t}^{(1)}$ for $t \geq T$.

Proof. By (A.2) and (2.3), we have

$\mathrm{d}\left|X_{t}^{(1)}-Y_{t}^{(1)}\right| \leq\left(K_{T}\left|X_{t}^{(1)}-Y_{t}^{(1)}\right|-\frac{2 K_{T}\left|x^{(1)}-y^{(1)}\right| \mathrm{e}^{-K_{T} t}}{1-\mathrm{e}^{-2 K_{T} T}}\right) \mathrm{d} t, \quad t \in\left[0, \tau_{1}\right) \cap[0, T]$. 
Then

$$
\left|X_{t}^{(1)}-Y_{t}^{(1)}\right| \leq \frac{\mathrm{e}^{-K_{T} t}-\mathrm{e}^{-K_{T}(2 T-t)}}{1-\mathrm{e}^{-2 K_{T} T}}\left|x^{(1)}-y^{(1)}\right|, \quad t \in\left[0, \tau_{1}\right) \cap[0, T] .
$$

This implies $\tau_{1} \leq T$ and also (2.4), since $X_{t}^{(1)}=Y_{t}^{(1)}$ for $t \geq \tau_{1}$.

To formulate (2.2) as the first equation in (1.1), we let

$$
\tilde{B}_{t}^{(1)}=B_{t}^{(1)}-\int_{0}^{t} \xi^{(1)}(s) \mathrm{d} s, \quad \xi^{(1)}(t):=\sigma^{(1)}(t)^{-1} v_{t}^{(1)}, \quad t \geq 0 .
$$

From (A.1) and (2.3) we see that $\xi^{(1)}(s)$ is bounded and adapted. So, by the Girsanov theorem, $\tilde{B}_{t}$ is an $m$-dimensional Brownian motion under the probability measure $\mathbb{Q}^{(1)}:=R_{1}(T) \mathbb{P}$, where

$$
R_{1}(t):=\exp \left[\int_{0}^{t}\left\langle\xi^{(1)}(s), \mathrm{d} B_{s}^{(1)}\right\rangle-\frac{1}{2} \int_{0}^{t}\left|\xi^{(1)}(s)\right|^{2} \mathrm{~d} s\right], \quad t \geq 0
$$

is a martingale. Obviously, (2.2) can be formulated as

$$
\mathrm{d} Y_{t}^{(1)}=b^{(1)}\left(t, Y_{t}^{(1)}\right) \mathrm{d} t+\sigma^{(1)}(t) \mathrm{d} \tilde{B}_{t}^{(1)}, \quad Y_{0}^{(1)}=y^{(1)} .
$$

As shown in (2.1), for the log-Harnack inequality we need to estimate the entropy of $R_{1}:=R_{1}(T)$.

Proposition 2.2. Let $R_{1}=R_{1}(T)$. Then

$$
\mathbb{E}\left\{R_{1} \log R_{1}\right\} \leq \frac{K_{T}\left|x^{(1)}-y^{(1)}\right|^{2}}{\lambda_{T}^{2}\left(1-\mathrm{e}^{-2 K_{T} T}\right)} .
$$

Proof. By $\tau_{1} \leq T,($ A.1), and (2.3), we have

$$
\int_{0}^{T}\left|\sigma^{(1)}(t)^{-1} v_{t}^{(1)}\right|^{2} \mathrm{~d} t \leq \frac{2 K_{T}\left|x^{(1)}-y^{(1)}\right|^{2}}{\lambda_{T}^{2}\left(1-\mathrm{e}^{-2 K_{T} T}\right)} .
$$

Then, it follows from (2.2) and the definition of $R_{1}$ that

$$
\begin{aligned}
\mathbb{E}\left\{R_{1} \log R_{1}\right\} & =\mathbb{E}_{\mathbb{Q}^{(1)}} \log R_{1} \\
& =\frac{1}{2} \mathbb{E}_{\mathbb{Q}^{(1)}} \int_{0}^{T}\left|\sigma^{(1)}(t)^{-1} v_{t}^{(1)}\right|^{2} \mathrm{~d} t \leq \frac{K_{T}\left|x^{(1)}-y^{(1)}\right|^{2}}{\lambda_{T}^{2}\left(1-\mathrm{e}^{-2 K_{T} T}\right)} .
\end{aligned}
$$

2.1.2. Construction of $\boldsymbol{Y}_{t}^{(2)}$. Consider the equation

$$
\mathrm{d} Y_{t}^{(2)}=b^{(2)}\left(t, Y_{t}\right) \mathrm{d} t+\sigma^{(2)}\left(t, Y_{t}^{(1)}\right) \mathrm{d} B_{t}-v_{t}^{(2)} \mathrm{d} t, \quad Y_{0}^{(2)}=y^{(2)},
$$

where

$$
v_{t}^{(2)}:=\frac{2 \Theta_{2 T}\left|X_{T}^{(2)}-Y_{T}^{(2)}\right| \mathrm{e}^{-\Theta_{2 T} t}\left(Y_{t}^{(2)}-X_{t}^{(2)}\right)}{\left(\mathrm{e}^{-2 \Theta_{2 T} T}-\mathrm{e}^{-4 \Theta_{2 T} T}\right)\left|X_{t}^{(2)}-Y_{t}^{(2)}\right|} 1_{\left\{t \geq T, X_{t}^{(2)} \neq Y_{t}^{(2)}\right\}}, \quad t \geq 0 .
$$


As $Y_{t}^{(1)}$ is now fixed, it is easy to see that (2.8) has a unique solution before the time

$$
\tau_{2}:=\inf \left\{t \geq T: X_{t}^{(2)}=Y_{t}^{(2)}\right\} .
$$

Letting $Y_{t}^{(2)}=X_{t}^{(2)}$ for $t \geq \tau_{2}$, we see that $\left(Y_{t}^{(2)}\right)_{t \geq 0}$ solves the equation (2.8). Thus,

$$
v_{t}^{(2)}=\frac{2 \Theta_{2 T}\left|X_{T}^{(2)}-Y_{T}^{(2)}\right| \mathrm{e}^{-\Theta_{2 T} t}\left(Y_{t}^{(2)}-X_{t}^{(2)}\right)}{\left(\mathrm{e}^{-2 \Theta_{2 T} T}-\mathrm{e}^{-4 \Theta_{2 T} T}\right)\left|X_{t}^{(2)}-Y_{t}^{(2)}\right|} 1_{\left[T, \tau_{2}\right)}(t), \quad t \geq 0 .
$$

Proposition 2.3. For any $t \geq T$,

$$
\left|X_{t}^{(2)}-Y_{t}^{(2)}\right| \leq \frac{\mathrm{e}^{-\Theta_{2 T}(t-T)}-\mathrm{e}^{-\Theta_{2 T}(3 T-t)}}{1-\mathrm{e}^{-2 \Theta_{2 T} T}}\left|X_{T}^{(2)}-Y_{T}^{(2)}\right| 1_{[T, 2 T]}(t) .
$$

Proof. Since $\varphi .(0)=0$ and $X_{t}^{(1)}=Y_{t}^{(1)}$ for $t \geq T$, by (A.3), (2.9), and Itô's formula we obtain

$$
\mathrm{d}\left|X_{t}^{(2)}-Y_{t}^{(2)}\right| \leq\left(\Theta_{2 T}\left|X_{t}^{(2)}-Y_{t}^{(2)}\right|-\frac{2 \Theta_{2 T}\left|X_{T}^{(2)}-Y_{T}^{(2)}\right| \mathrm{e}^{-\Theta_{2 T} t}}{\mathrm{e}^{-2 \Theta_{2 T}}-\mathrm{e}^{-4 \Theta_{2 T} T}}\right) \mathrm{d} t
$$

for $t \in\left[T, \tau_{2}\right) \cap[T, 2 T]$. This implies (2.10) for $t \in\left[T, \tau_{2}\right) \cap[T, 2 T]$. Therefore, $\tau_{2} \leq 2 T$ and (2.10) holds for all $t \geq T$.

To formulate (2.8) as the second equation in (1.1), we need to make use of the Girsanov theorem to get rid of the additional drift. To this end, let

$$
\xi^{(2)}(s)=\sigma^{(2)}\left(s, Y_{s}^{(1)}\right)^{-1} v_{s}^{(2)}, \quad s \in[T, 2 T],
$$

and

$$
R_{2}(t)=\exp \left[\int_{T}^{t}\left\langle\xi^{(2)}(s), \mathrm{d} B_{s}^{(2)}\right\rangle-\frac{1}{2} \int_{T}^{t}\left|\xi^{(2)}(s)\right|^{2} \mathrm{~d} s\right], \quad t \in[T, 2 T] .
$$

Since $B_{t}^{(2)}$ is independent of $\mathcal{F}^{(1)}$, the following result ensures that $\left\{R_{2}(t)\right\}_{t \in[T, 2 T]}$ is a uniformly integrable $\mathcal{F}_{t}^{(2)}$-martingale under $\mathbb{P}\left(\cdot \mid \mathcal{F}^{(1)}\right)$.

Proposition 2.4. Under $\mathbb{P}\left(\cdot \mid \mathcal{F}^{(1)}\right),\left\{R_{2}(t)\right\}_{t \in[T, 2 T]}$ is an $\mathcal{F}_{t}^{(2)}$-martingale, and $R_{2}:=R_{2}(2 T)$ satisfies

$$
\begin{aligned}
\mathbb{E}_{\mathbb{P}(\cdot \mid \mathcal{F}(1)}\left\{R_{2} \log R_{2}\right\} \leq & \left(\int_{T}^{2 T} \frac{2 \Theta_{2 T}^{2} \mathrm{e}^{-2 \Theta_{2 T} t}\left\|\sigma^{(2)}\left(t, Y_{t}^{(1)}\right)^{-1}\right\|^{2}}{\left(\mathrm{e}^{-2 \Theta_{2 T} T}-\mathrm{e}^{-4 \Theta_{2 T} T}\right)^{2}} \mathrm{~d} t\right) \\
& \times\left(\mathrm{e}^{2 \Theta_{T} T}\left|x^{(2)}-y^{(2)}\right|^{2}+\frac{\mathrm{e}^{2 \Theta_{T} T}-1}{\Theta_{T}} \varphi_{T}\left(\left|x^{(1)}-y^{(1)}\right|^{2}\right)\right) \\
& \times \sup _{t \in[0, T]} h\left(\left|Y_{t}^{(1)}\right|+\left|x^{(1)}-y^{(1)}\right|\right) .
\end{aligned}
$$


Proof. We use an approximation argument. Let $\xi_{n}^{(2)}(s)=\xi^{(2)}(s) 1_{\left\{\left|\xi^{(2)}(s)\right| \leq n\right\}}$, and

$$
R_{2, n}(t)=\exp \left[\int_{T}^{t}\left\langle\xi_{n}^{(2)}(s), \mathrm{d} B_{s}^{(2)}\right\rangle-\frac{1}{2} \int_{T}^{t}\left|\xi_{n}^{(2)}(s)\right|^{2} \mathrm{~d} s\right], \quad n \geq 1, t \in[T, 2 T] .
$$

Then $\left\{R_{2, n}(t)\right\}_{t \in[T, 2 T]}$ is an $\mathcal{F}_{t}^{(2)}$-martingale under $\mathbb{P}\left(\cdot \mid \mathcal{F}^{(1)}\right)$. So, it remains to show that

$\mathbb{E}_{\mathbb{P}\left(\cdot \mid \mathcal{F}^{(1)}\right)}\left\{R_{2, n} \log R_{2, n}\right\}(t) \leq\left(\int_{T}^{2 T} \frac{2 \Theta_{2 T}^{2} \mathrm{e}^{-2 \Theta_{2 T} t}\left\|\sigma^{(2)}\left(t, Y_{t}^{(1)}\right)^{-1}\right\|^{2}}{\left(\mathrm{e}^{-2 \Theta_{2 T} T}-\mathrm{e}^{-4 \Theta_{2 T} T}\right)^{2}} \mathrm{~d} t\right)$

$$
\begin{aligned}
& \times\left(\mathrm{e}^{2 \Theta_{T} T}\left|x^{(2)}-y^{(2)}\right|^{2}+\frac{\mathrm{e}^{2 \Theta_{T} T}-1}{\Theta_{T}} \varphi_{T}\left(\left|x^{(1)}-y^{(1)}\right|^{2}\right)\right) \\
& \times \sup _{t \in[0, T]} h\left(\left|Y_{t}^{(1)}\right|+\left|x^{(1)}-y^{(1)}\right|\right)
\end{aligned}
$$

holds for all $t \in[T, 2 T]$ and $n \geq 1$. Let $\mathbb{Q}_{2, n}=R_{2, n}(2 T) \mathbb{P}\left(\cdot \mid \mathcal{F}^{(1)}\right)$. By the Girsanov theorem, under $\mathbb{Q}_{2, n}$ the process

$$
\tilde{B}_{t}^{(2)}:=B_{t}^{(2)}-\int_{T}^{T \vee t} \xi_{n}^{(2)}(s) \mathrm{d} s, \quad t \in[0,2 T],
$$

is a $d$-dimensional Brownian motion. Then, by the definition of $\xi_{n}^{(2)}(s)$ and $(2.9)$, we have

(2.13) $\mathbb{E}_{\mathbb{P}(\cdot \mid \mathcal{F}(1))}\left\{R_{2, n} \log R_{2, n}\right\}(2 T)=\mathbb{E}_{\mathbb{Q}_{2, n}} \log R_{2, n}(2 T)=\frac{1}{2} \int_{T}^{2 T} \mathbb{E}_{\mathbb{Q}_{2, n}}\left|\xi_{n}^{(2)}(s)\right|^{2} \mathrm{~d} s$ $\leq\left(\int_{T}^{2 T} \frac{2 \Theta_{2 T}^{2} \mathrm{e}^{-2 \Theta_{2 T} t}\left\|\sigma^{(2)}\left(t, Y_{t}^{(1)}\right)^{-1}\right\|^{2}}{\left(\mathrm{e}^{-2 \Theta_{2 T} T}-\mathrm{e}^{-4 \Theta_{2 T} T}\right)^{2}} \mathrm{~d} t\right) \cdot \mathbb{E}_{\mathbb{P}\left(\cdot \mid \mathcal{F}^{(1)}\right)}\left\{R_{2, n}(2 T)\left|X_{T}^{(2)}-Y_{T}^{(2)}\right|^{2}\right\}$.

Since $\left\{R_{2, n}(t)\right\}_{t \in[T, 2 T]}$ is an $\mathcal{F}_{t}^{(2)}$-martingale under $P\left(\cdot \mid \mathcal{F}^{(1)}\right)$, and $R_{2, n}(T)=1$,

$$
\mathbb{E}_{\mathbb{P}\left(\cdot \mid \mathcal{F}^{(1)}\right)}\left\{R_{2, n}(2 T)\left|X_{T}^{(2)}-Y_{T}^{(2)}\right|^{2}\right\}=\mathbb{E}_{\mathbb{P}\left(\cdot \mid \mathcal{F}^{(1)}\right)}\left|X_{T}^{(2)}-Y_{T}^{(2)}\right|^{2} .
$$

Finally, by (A.3), (2.4), and Itô's formula, we obtain

$$
\begin{aligned}
\mathrm{d} \mid X_{t}^{(2)} & -\left.Y_{t}^{(2)}\right|^{2} \leq 2\left\langle X_{t}^{(2)}-Y_{t}^{(2)},\left\{\sigma^{(2)}\left(t, X_{t}^{(1)}\right)-\sigma^{(2)}\left(t, Y_{t}^{(1)}\right)\right\} \mathrm{d} B_{t}^{(2)}\right\rangle \\
& +2\left\{\Theta_{T}\left|X_{t}^{(2)}-Y_{t}^{(2)}\right|^{2}+\varphi_{T}\left(\left|x^{(1)}-y^{(1)}\right|^{2}\right) h\left(\left|Y_{t}^{(1)}\right|+\left|x^{(1)}-y^{(1)}\right|\right)\right\} \mathrm{d} t
\end{aligned}
$$

for $t \in[T, 2 T]$. Since $h \geq 1$, this implies

$$
\begin{aligned}
\mathbb{E}_{\mathbb{P}(\cdot \mid \mathcal{F}(1))}\left|X_{T}^{(2)}-Y_{T}^{(2)}\right|^{2} \leq & \sup _{t \in[0, T]} h\left(\left|Y_{t}^{(1)}\right|+\left|x^{(1)}-y^{(1)}\right|\right) \\
& \times\left(\mathrm{e}^{2 \Theta_{T} T}\left|x^{(2)}-y^{(2)}\right|^{2}+\frac{\mathrm{e}^{2 \Theta_{T} T}-1}{\Theta_{T}} \varphi_{T}\left(\left|x^{(1)}-y^{(1)}\right|^{2}\right)\right) .
\end{aligned}
$$

Combining this with (2.13) and (2.14), we prove (2.12). 
Proof of Theorem 1.1. Let $X_{t}=\left(X_{t}^{(1)}, X_{t}^{(2)}\right)$ and $Y_{t}=\left(Y_{t}^{(1)}, Y_{t}^{(2)}\right)$ be as constructed above. Let $R=R_{1} R_{2}$. By Propositions 2.1, 2.2, 2.3, and 2.4, we have $X_{2 T}=Y_{2 T}, \mathbb{E}_{\mathbb{P}\left(\cdot \mid \mathcal{F}^{(1)}\right)} R_{2}=1$, and, noting that the distribution of $Y^{(1)}$ under $R_{1} \mathbb{P}$ coincides with that of $X^{(1)}\left(y^{(1)}\right)$ under $\mathbb{P}$,

$$
\begin{aligned}
\mathbb{E}\{R \log R\}= & \mathbb{E}\left\{\left(R_{1} \log R_{1}\right) \mathbb{E}_{\mathbb{P}(\cdot \mid \mathcal{F}(1)} R_{2}\right\}+\mathbb{E}\left\{R_{1} \mathbb{E}_{\mathbb{P}(\cdot \mid \mathcal{F}(1)}\left(R_{2} \log R_{2}\right)\right\} \\
\leq & \frac{K_{T}\left|x^{(1)}-y^{(1)}\right|^{2}}{\lambda_{T}^{2}\left(1-\mathrm{e}^{-2 K_{T} T}\right)}+\mathbb{E}^{y^{(1)}}\left\{\left(\int_{T}^{2 T} \frac{2 \Theta_{2 T}^{2} \mathrm{e}^{-2 \Theta_{2 T} t}\left\|\sigma^{(2)}\left(t, X_{t}^{(1)}\right)^{-1}\right\|^{2}}{\left(\mathrm{e}^{-2 \Theta_{2 T} T}-\mathrm{e}^{-4 \Theta_{2 T} T}\right)^{2}} \mathrm{~d} t\right)\right. \\
& \times\left(\mathrm{e}^{2 \Theta_{T} T}\left|x^{(2)}-y^{(2)}\right|^{2}+\frac{\mathrm{e}^{2 \Theta_{T}^{T}}-1}{\Theta_{T}} \varphi_{T}\left(\left|x^{(1)}-y^{(1)}\right|^{2}\right)\right) \\
& \left.\times \sup _{t \in[0, T]} h\left(\left|X_{t}^{(1)}\right|+\left|x^{(1)}-y^{(1)}\right|\right)\right\} \\
\leq & \frac{K_{T}\left|x^{(1)}-y^{(1)}\right|^{2}}{\lambda_{T}^{2}\left(1-\mathrm{e}^{-2 K_{T} T}\right)}+\frac{\Theta_{2 T} \mathrm{e}^{2 \Theta_{T} T} \psi_{T}\left(x^{(1)}, y^{(1)}\right)}{\mathrm{e}^{-2 \Theta_{2 T} T}-\mathrm{e}^{-4 \Theta_{2 T} T}} \\
& \times\left\{\left|x^{(2)}-y^{(2)}\right|^{2}+\frac{1-\mathrm{e}^{-2 \Theta_{T} T}}{\Theta_{T}} \varphi_{T}\left(\left|x^{(1)}-y^{(1)}\right|^{2}\right)\right\} .
\end{aligned}
$$

Therefore, the desired log-Harnack inequality follows from (2.1), since under the probability measure $\mathbb{Q}:=R \mathbb{P}$

$$
\tilde{B}_{t}:=B_{t}+\int_{0}^{t}\left(\xi^{(1)}(s), \xi^{(2)}(s)\right) \mathrm{d} s, \quad t \geq 0,
$$

is a Brownian motion on $\mathbb{R}^{m+d}$, and $Y_{t}$ with $Y_{0}=y$ solves the equation

$$
\left\{\begin{array}{l}
\mathrm{d} Y_{t}^{(1)}=b^{(1)}\left(t, Y_{t}^{(1)}\right) \mathrm{d} t+\sigma^{(1)}(t) \mathrm{d} \tilde{B}_{t}^{(1)}, \\
\mathrm{d} \tilde{Y}_{t}^{(2)}=b^{(2)}\left(t, Y_{t}\right) \mathrm{d} t+\sigma^{(2)}\left(t, Y_{t}^{(1)}\right) \mathrm{d} \tilde{B}_{t}^{(2)},
\end{array}\right.
$$

so that $P_{2 T} f(y)=\mathbb{E}_{\mathbb{Q}} f\left(Y_{2 T}\right)=\mathbb{E}\left\{R f\left(Y_{2 T}\right)\right\}=\mathbb{E}\left\{R f\left(X_{2 T}\right)\right\}$.

\section{Proofs of Theorem 1.2 and Corollary 1.3}

Proof of Theorem 1.2. Let $X_{t}=\left(X_{t}^{(1)}, X_{t}^{(2)}\right)$ and $Y_{t}^{(1)}$ be constructed as in the previous section. We now modify the construction of $Y_{t}^{(2)}$ to take into account the condition $\Psi_{T}<\infty$. Let

$$
\eta_{t}=\sigma^{(2)}\left(t, Y_{t}^{(1)}\right)^{*} \mathrm{e}^{A^{*}(T-t)} Q_{T}^{-1}\left(Y_{T}^{(2)}-X_{T}^{(2)}\right) 1_{[T, 2 T]}(t), \quad t \geq 0 .
$$

Let $Y_{t}^{(2)}$ solve the equation

$$
\mathrm{d} Y_{t}^{(2)}=b^{(2)}\left(t, Y_{t}\right) \mathrm{d} t+\sigma^{(2)}\left(t, Y_{t}^{(1)}\right)\left\{\mathrm{d} B_{t}^{(2)}-\eta_{t} \mathrm{~d} t\right\}, Y_{0}^{(2)}=y^{(2)} .
$$

Since under $\mathbb{P}\left(\cdot \mid \mathcal{F}^{(1)}\right)$ the processes $X_{t}^{(1)}$ and $Y_{t}^{(1)}$ are fixed and $B_{t}^{(2)}$ is a $d$ dimensional Brownian motion, by (A.3) this equation has a unique solution. Since 
$X_{t}^{(1)}=Y_{t}^{(1)}$ for $t \geq T$, for the present $b^{(2)}$ we have $b^{(2)}\left(t, X_{t}\right)-b^{(2)}\left(t, Y_{t}\right)=$ $A\left(X_{t}^{(2)}-Y_{t}^{(2)}\right)$ for $t \geq T$. So,

$$
X_{2 T}^{(2)}-Y_{2 T}^{(2)}=\mathrm{e}^{A T}\left(X_{T}^{(2)}-Y_{T}^{(2)}\right)+\int_{T}^{2 T} \mathrm{e}^{A(2 T-t)} \sigma^{(2)}\left(t, Y_{t}^{(1)}\right) \eta_{t} \mathrm{~d} t=0
$$

as $Y_{t}^{(1)}=X_{t}^{(1)}$ for $t \geq T$. Therefore, $X_{2 T}=Y_{2 T}$. Moreover, let

$$
\tilde{R}_{2}=\exp \left[\int_{T}^{2 T}\left\langle\eta_{t}, \mathrm{~d} B_{t}^{(2)}\right\rangle-\frac{1}{2} \int_{T}^{2 T}\left|\eta_{t}\right|^{2} \mathrm{~d} t\right] .
$$

Following the proof of Proposition 2.4 and using (3.1), we obtain

$$
\begin{aligned}
\mathbb{E}_{\mathbb{P}(\cdot \mid \mathcal{F}(1)}\{ & \left\{\tilde{R}_{2} \log \tilde{R}_{2}\right\}=\frac{1}{2} \int_{T}^{2 T} \mathbb{E}_{\tilde{R}_{2} \mathbb{P}\left(\cdot \mid \mathcal{F}^{(1)}\right)}\left|\eta_{t}\right|^{2} \mathrm{~d} t \\
\leq & \frac{\theta_{T}}{2}\left(\mathbb{E}_{\mathbb{P}(\cdot \mid \mathcal{F}(1)}\left\|X_{T}^{(2)}-Y_{T}^{(2)}\right\|^{2}\right)\left\|Q_{T}^{-1}\right\|^{2} \int_{T}^{2 T}\left\|\sigma^{(2)}\left(t, Y_{t}^{(1)}\right)\right\|^{2} \mathrm{~d} t \\
\leq & \frac{\theta_{T}\left\|Q_{T}^{-1}\right\|^{2}}{2}\left\{\mathrm{e}^{2 \Theta_{T} T}\left|x^{(2)}-y^{(2)}\right|^{2}+\frac{\mathrm{e}^{2 \Theta_{T} T}-1}{\Theta_{T}} \varphi_{T}\left(\left|x^{(1)}-y^{(1)}\right|^{2}\right)\right\} \\
& \times \sup _{t \in[0, T]} h\left(\left|Y_{t}^{(1)}\right|+\left|x^{(1)}-y^{(1)}\right|\right) \int_{T}^{2 T}\left\|\sigma^{(2)}\left(t, Y_{t}^{(1)}\right)\right\|^{2} \mathrm{~d} t .
\end{aligned}
$$

Repeating the proof of Theorem 1.1 and using this inequality instead of (2.11), we obtain

$$
\begin{aligned}
& \mathbb{E}\left\{\left(R_{1} \tilde{R}_{2}\right) \log \left(R_{1} \tilde{R}_{2}\right)\right\} \leq \frac{K_{T}\left|x^{(1)}-y^{(1)}\right|^{2}}{\lambda_{T}^{2}\left(1-\mathrm{e}^{-2 K_{T} T}\right)} \\
& (3.2)+\frac{\theta_{T} \mathrm{e}^{2 \Theta_{T} T} \Psi_{T}\left(x^{(1)}, y^{(1)}\right)}{2}\left\{\left|x^{(2)}-y^{(2)}\right|^{2}+\frac{1-\mathrm{e}^{-2 \Theta_{T} T}}{\Theta_{T}} \varphi_{T}\left(\left|x^{(1)}-y^{(1)}\right|^{2}\right)\right\} .
\end{aligned}
$$

Since $B_{t}^{(2)}$ is a $d$-dimensional Brownian motion under $\mathbb{P}\left(\cdot \mid \mathcal{F}^{(1)}\right)$, by the Girsanov theorem, under $\tilde{R}_{2} \mathbb{P}\left(\cdot \mid \mathcal{F}^{(1)}\right)$ the process

$$
\tilde{B}_{t}^{(2)}:=B_{t}^{(2)}-\int_{T}^{t} \eta_{s} \mathrm{~d} s, \quad t \in[T, 2 T]
$$

is a $d$-dimensional Brownian motion. Noting that

$$
Y_{t}^{(2)}=Y_{T}^{(2)}+\int_{T}^{t} b^{(2)}\left(s, Y_{s}\right) \mathrm{d} s+\int_{T}^{t} \sigma^{(2)}\left(s, Y_{s}^{(1)}\right) \mathrm{d} \tilde{B}_{s}^{(2)}, \quad t \in[T, 2 T],
$$

we see that the distribution of $Y_{2 T}^{(2)}$ under $\tilde{R}_{2} \mathbb{P}\left(\cdot \mid \mathcal{F}^{(1)}\right)$ coincides with that of $\tilde{Y}_{2 T}^{(2)}$ under $\mathbb{P}\left(\cdot \mid \mathcal{F}^{(1)}\right)$, where

$$
\tilde{Y}_{t}^{(2)}= \begin{cases}Y_{t}^{(2)}, & \text { if } t \in[0, T], \\ Y_{T}^{(2)}+\int_{T}^{t} b^{(2)}\left(s, Y_{s}\right) \mathrm{d} s+\int_{T}^{t} \sigma^{(2)}\left(s, Y_{s}^{(1)}\right) \mathrm{d} B_{s}^{(2)}, & \text { if } t \in[T, 2 T] .\end{cases}
$$


Therefore,

$$
\mathbb{E}_{\mathbb{P}\left(\cdot \mid \mathcal{F}^{(1)}\right)}\left\{\tilde{R}_{2} \log f\left(Y_{2 T}\right)\right\}=\mathbb{E}_{\mathbb{P}\left(\cdot \mid \mathcal{F}^{(1)}\right)}\left\{\log f\left(Y_{2 T}^{(1)}, \tilde{Y}_{2 T}^{(2)}\right)\right\} .
$$

Combining this with $X_{2 T}=Y_{2 T}$, we obtain

$$
\begin{array}{r}
\mathbb{E}\left\{R_{1} \tilde{R}_{2} \log f\left(X_{2 T}\right)\right\}=\mathbb{E}\left\{R_{1} \tilde{R}_{2} \log f\left(Y_{2 T}\right)\right\}=\mathbb{E}\left(R_{1} \mathbb{E}_{\mathbb{P}\left(\cdot \mid \mathcal{F}^{(1)}\right)}\left\{\tilde{R}_{2} \log f\left(Y_{2 T}\right)\right\}\right) \\
=\mathbb{E}\left(R_{1} \mathbb{E}_{\mathbb{P}\left(\cdot \mid \mathcal{F}^{(1)}\right)}\left\{\log f\left(Y_{2 T}^{(1)}, \tilde{Y}_{2 T}^{(2)}\right)\right\}\right)=\mathbb{E}\left\{R_{1} \log f\left(Y_{2 T}^{(1)}, \tilde{Y}_{2 T}^{(2)}\right)\right\} .
\end{array}
$$

Moreover, since

$$
\tilde{B}_{t}^{(1)}=B_{t}^{(1)}-\int_{0}^{T \wedge t} \xi^{(1)}(s) \mathrm{d} s, \quad t \in[0,2 T],
$$

again by the Girsanov theorem, under $R_{1} \mathbb{P}$ the process $\left(\tilde{B}_{t}^{(1)}, B_{t}^{(2)}\right)_{t \in[0,2 T]}$ is a $(d+m)$-dimensional Brownian motion. Noting that $\left(Y_{t}^{(1)}, \tilde{Y}_{t}^{(2)}\right)$ solves the equation

$$
\begin{cases}\mathrm{d} Y_{t}^{(1)}=b^{(1)}\left(t, Y_{t}^{(1)}\right) \mathrm{d} t+\sigma^{(1)}(t) \mathrm{d} \tilde{B}_{t}^{(1)}, & Y_{0}^{(1)}=y^{(1)} \\ \mathrm{d} \tilde{Y}_{t}^{(2)}=b^{(2)}\left(t, Y_{t}^{(1)}, \tilde{Y}_{t}^{(2)}\right) \mathrm{d} t+\sigma^{(2)}\left(t, Y_{t}^{(1)}\right) \mathrm{d} B_{t}^{(2)}, & \tilde{Y}_{0}^{(2)}=y^{(2)}\end{cases}
$$

we conclude that the distribution of $\left(Y_{2 T}^{(1)}, \tilde{Y}_{2 T}^{(2)}\right)$ under $R_{1} \mathbb{P}$ coincides with that of $X_{2 T}(y)$ under $\mathbb{P}$. Therefore, it follows from (3.3) and the Young inequality that

$$
\begin{aligned}
P_{2 T} \log f(y) & =\mathbb{E}\left\{R_{1} \log f\left(Y_{2 T}^{(1)}, \tilde{Y}_{2 T}^{(2)}\right)\right\}=\mathbb{E}\left\{R_{1} \tilde{R}_{2} \log f\left(X_{2 T}\right)\right\} \\
& \leq \log P_{2 T} f(x)+\mathbb{E}\left\{\left(R_{1} \tilde{R}_{2}\right) \log \left(R_{1} \tilde{R}_{2}\right)\right\} .
\end{aligned}
$$

Combining this with (3.2) we complete the proof.

Proof of Corollary 1.3. It is easy to see that (A.1)-(A.3) hold for $\lambda=1, K=\Theta=0$, $\varphi(r)=r^{l \wedge 1}$, and $h(r)=c_{1} \vee r^{2(l-1)^{+}}$for some constant $c_{1} \geq 1$. Moreover,

$$
Q_{T}=I_{d \times d} \int_{T}^{2 T}\left|B_{t}^{(1)}+x^{(1)}\right|^{2 l} \mathrm{~d} t
$$

is invertible and

$$
\left\|Q_{T}^{-1}\right\|^{2} \int_{T}^{2 T}\left\|\sigma^{(2)}\left(X_{t}^{(1)}\right)\right\|^{2} \mathrm{~d} t=\frac{1}{\int_{T}^{2 T}\left|B_{t}^{(1)}+x^{(1)}\right|^{2 l} \mathrm{~d} t} .
$$

Then, using the fact that for any $r \geq 0$,

$$
\mathbb{E} \sup _{t \in[0, T]}\left|B_{t}^{(1)}+x^{(1)}\right|^{2 r} \leq c(r)\left(\left|x^{(1)}\right|^{2 r}+T^{r}\right)
$$

holds for some constant $c(r)>0$, and noting that Lemma 3.1 in [18] implies

$$
\begin{aligned}
& \mathbb{E}\left(\int_{T}^{2 T}\left|B_{t}^{(1)}+x^{(1)}\right|^{2 l} \mathrm{~d} t\right)^{-2} \\
& \quad=\mathbb{E}\left\{\mathbb{E}\left(\left(\int_{0}^{T}\left|\left(B_{T+t}^{(1)}-B_{T}^{(1)}\right)+\left(B_{T}^{(1)}+x^{(1)}\right)\right|^{2 l} \mathrm{~d} t\right)^{-2} \mid B_{T}^{(1)}\right)\right\} \leq \frac{C}{T^{2(l+1)}}
\end{aligned}
$$


for some constant $C>0$, we conclude that

$$
\begin{aligned}
\Psi_{T}\left(x^{(1)}, y^{(1)}\right) \leq & \left(\mathbb{E} \sup _{t \in[0, T]} h\left(\left|B_{t}^{(1)}+x^{(1)}\right|+\left|x^{(1)}-y^{(1)}\right|\right)^{2}\right)^{1 / 2} \\
& \times\left(\mathbb{E}\left(\int_{T}^{2 T}\left|B_{t}^{(1)}+x^{(1)}\right|^{2 l} \mathrm{~d} t\right)^{-2}\right)^{1 / 2} \\
\leq & \frac{c}{T^{l+1}}\left(\left|x^{(1)}\right|^{2(l-1)^{+}}+\left|y^{(1)}\right|^{2(l-1)^{+}}+T^{(l-1)^{+}}\right)
\end{aligned}
$$

holds for some constant $c>0$. Therefore, the desired log-Harnack inequality follows from Theorem 1.2.

\section{References}

[1] Arnaudon, M., Thalmaier, A. And Wang, F. Y.: Harnack inequality and heat kernel estimates on manifolds with curvature unbounded below. Bull. Sci. Math. 130 (2006), no. 3, 223-233.

[2] Bakry, D., Baudoin, F., Bonnefont, M. and Chafaї, D.: On gradient bounds for the heat kernel on the Heisenberg group. J. Funct. Anal. 255 (2008), no. 8, 1905-1938.

[3] Baudoin, F. And Bonnefont, M.: Log-Sobolev inequalities for subelliptic operators satisfying a generalized curvature dimension inequality. J. Funct. Anal. 262 (2012), no. 6, 2646-2676.

[4] Baudoin, F., Bonnefont, M. And Garofalo, N.: A sub-Riemannian curvaturedimension inequality, volume doubling property and the Poincaré inequality. Math. Ann. 358 (2014), no. 3-4, 833-860.

[5] Baudoin, F., Gordina, M. And Melcher, T.: Quasi-invariance for heat kernel measures on sub-Riemannian infinite-dimensional Heisenberg groups. Trans. Amer. Math. Soc. 365 (2013), no. 8, 4313-4350.

[6] Driver, B. K. And Melcher, T.: Hypoelliptic heat kernel inequalities on the Heisenberg group. J. Funct. Anal. 221 (2005), no. 2, 340-365.

[7] Guillin, A. And WAng F. Y.: Degenerate Fokker-Planck equations: Bismut formula, gradient estimate and Harnack inequality. J. Differential Equations 253 (2012), no. 1, 20-40.

[8] LI, H. Q.: Estimation optimale du gradient du semi-groupe de la chaleur sur le groupe de Heisenberg. J. Funct. Anal. 236 (2006), no. 2, 369-394.

[9] Liu, W.: Harnack inequality and applications for stochastic evolution equations with monotone drifts. J. Evol. Equ. 9 (2009), no. 4, 747-770.

[10] Liu, W. AND WANG, F. Y.: Harnack inequality and strong Feller property for stochastic fast-diffusion equations. J. Math. Anal. Appl. 342 (2008), no. 1, 651-662.

[11] OuYAnG, S.X.: Harnack inequalities and applications for multivalued stochastic evolution equations. Inf. Dimens. Anal. Quantum Probab. Relat. Top. 14 (2011), no. 2, 261-278. 
[12] Ouyang, S.X., Röckner, M. And Wang, F. Y.: Harnack inequalities and applications for Ornstein-Uhlenbeck semigroups with jump. Potential Anal. 36 (2012), no. 2, 301-315.

[13] Röckner, M. And WAng, F. Y.: Log-Harnack inequality for stochastic differential equations in Hilbert spaces and its consequences. Infin. Dimens. Anal. Quantum Probab. Relat. Top. 13 (2010), no. 1, 27-37.

[14] WANG, F. Y.: Logarithmic Sobolev inequalities on noncompact Riemannian manifolds. Probab. Theory Related Fields 109 (1997), no. 3, 417-424.

[15] WANG, F. Y.: Harnack inequalities on manifolds with boundary and applications. J. Math. Pures Appl. (9) 94 (2010), no. 3, 304-321.

[16] WAng, F. Y.: Harnack inequality for SDE with multiplicative noise and extension to Neumann semigroup on nonconvex manifolds. Ann. Probab. 39 (2011), no. 4, 1449-1467.

[17] Wang, F. Y.: Derivative formula and gradient estimates for Gruschin type semigroups. J. Theoret. Probab. 27 (2014), no. 1, 30-49.

[18] Wang, F. Y.: Analysis for diffusion processes on Riemannian manifolds., Chapter 5. World Scientific, Singapore, 2013.

[19] Wang, F. Y., Wu, J. L. And Xu, L.: Log-Harnack inequality for stochastic Burgers equations and applications. J. Math. Anal. Appl. 384 (2011), no. 1, 151-159.

[20] Wang, F. Y. And YuAN, C.: Harnack inequalities for functional SDEs with multiplicative noise and applications. Stochastic Process. Appl. 121 (2011), no. 11, $2692-2710$

[21] Wang, F. Y. And Xu, L.: Derivative formula and applications for hyperdissipative stochastic Navier-Stokes/Burgers equations. Infin. Dimens. Anal. Quantum Probab. Relat. Top. 15 (2012), no. 3, 1250020, 19 pp.

[22] Wang, F. Y. And Zhang, X. C.: Derivative formula and applications for degenerate diffusion semigroups. J. Math. Pures Appl. (9) 99 (2013), no. 6, 726-740.

[23] XU, L.: A modified log-Harnack inequality and asymptotically strong Feller property. J. Evol. Equ. 11 (2011), no. 4, 925-942.

[24] Zhang, X. C.: Stochastic flows and Bismut formulas for stochastic Hamiltonian systems. Stochastic Process. Appl. 120 (2010), no. 10, 1929-1949.

Received June 6, 2012.

Feng-Yu Wang: School of Mathematical Sciences, Beijing Normal University, Beijing 100875, China; Department of Mathematics, Swansea University, Singleton Park, SA2 8PP, Swansea, UK.

E-mail: wangfy@bnu.edu.cn; F.Y.Wang@swansea.ac.uk

Linu Xu: Department of Mathematics, Faculty of Science and Technology, University of Macau, Av. Padre Tomas Pereira, Macau, China.

E-mail: lihuxu@umac.mo

Supported in part by NNSFC(11131003), SRFDP, the Laboratory of Mathematical and Complex Systems and the Fundamental Research Funds for the Central Universities. 